\title{
Disease-specific impairment of the quality of life in adult patients with chronic spontaneous urticaria
}

\author{
Won-Sun Choi, Eun-Soo Lim, Ga-Young Ban, Ji-Hye Kim, Yoo-Seob Shin, Hae-Sim Park, and \\ Young-Min Ye
}

Department of Allergy and Clinical Immunology, Ajou University School of Medicine, Suwon, Korea

Received: June 29, 2015

Revised : August 24, 2015

Accepted: September 17, 2015

\section{Correspondence to}

Young-Min Ye, M.D.

Department of Allergy and Clinical Immunology, Ajou University School of Medicine, 164 World cup-ro, Yeongtong-gu, Suwon 16499, Korea

Tel: +82-31-219-4262

Fax: +82-31-219-4265

E-mail:ye9oo7@ajou.ac.kr
Background/Aims: Chronic urticaria (CU) is a common skin disorder characterized by wheals and pruritus lasting more than 6 weeks. Due to its long duration and changeable symptoms, the quality of life (QOL) of patients with $\mathrm{CU}$ can be impaired substantially. We evaluated the CU-QOL, a previously validated CU-specific QOL measure, and investigated factors influencing QOL in chronic spontaneous urticaria (CSU) patients.

Methods: A hospital-based cross-sectional study was performed on 390 adult patients diagnosed with CSU from March 2009 to December 2012 at the Allergy and Clinical Immunology Clinic at Ajou University Hospital. The CU-QOL questionnaire, urticaria activity score (UAS), combined angioedema, and serum total immunoglobulin E (IgE) levels were investigated.

Results: The average CU-QOL score obtained from the questionnaire was 70.6 (of 100 points). The CU-QOL scores correlated significantly with the UAS, particularly with the 15-point UAS (UAS-15; coefficient $-0.532, p<0.01$ ) rather than the 6 -point UAS $(-0.502, p<0.01)$. The patients presenting with angioedema and urticaria had poorer scores in the urticaria symptom domain than those with urticaria alone (37.4 vs. 46.9, $p=0.004)$. Log-transformed serum total IgE levels correlated significantly with CU-QOL $(-0.131, p<0.05)$. Multivariate regression models indicated that severe CU (UAS-15 score $\geq 13$ ), $\log$ (total IgE), and the presence of angioedema were significant predictors of impaired CU-QOL ( $<85$ points).

Conclusions: CU has a substantial negative impact on QOL. The assessment of UAS-15, total IgE, and the presence of angioedema can be useful to predict QOL of the patients with CSU.

Keywords: Urticaria; Chronic; Quality of life; Angioedema; Immunoglobulin E

\section{INTRODUCTION}

Chronic urticaria (CU) is a common skin disorder, defined by recurrent wheals and pruritus, with or without angioedema for at least 6 weeks [1]. CU accounts for $25 \%$ of urticaria, and the prevalence of CU is $0.1 \%$ to $0.6 \%$ [2]. In acute urticaria (AU) possible eliciting factors, such as drug or food, are often identified by detailed patient his- tory taking. The course of AU is self-limited, by avoiding the offending factors, and is characterized by rapid responses to symptomatic treatment. However, about $80 \%$ patients with CU, the underlying cause is not identified and its pathogenesis is not clearly established $[1,3]$. These patients whose urticaria occurs without obvious extrinsic stimuli are diagnosed with chronic spontaneous urticaria (CSU). 
Urticaria is a mast cell-driven disease and histamine is considered to be the key mediator. Degranulation of skin mast cells by heterogeneous activating signals results in sensory nerve activation, vasodilation, plasma extravasation, and cell recruitment to urticarial lesions [3]. Many studies have shown that about $50 \%$ of CU is related to autoimmunity $[2,3]$. Elevated histamine releasability from skin mast cells and skin infiltration of blood basophils have also been noted in the pathogenesis of CU [3].

Health-related quality of life (HR-QOL) in CU can be impaired substantially due to its unpredictable symptoms and long-term nature. In a previous study, the impairment of QOL in CU was comparable with that of patients with coronary artery disease awaiting coronary artery bypass grafting [4]. Additionally, recent studies have shown psychological stress and emotional disturbances to be common in patients with CU [5-7]. Ozkan et al. [8] showed that $60 \%$ of CU patients had a psychiatric diagnosis and their QOL was reduced.

In most cases, the evaluation of CU has focused on clinical symptoms; however, understanding and dealing with QOL in CU patients is also necessary to improve patient management, address economic costs, and further clinical research. Recent guidelines recommended the use of the urticaria activity score (UAS) as a tool for assessing urticaria disease activity, and the CU-QOL questionnaire [9], which was the first measurement device developed in Italy to assess disease-specific QOL in patients with CU [2]. However, HR-QOL generally depends on the disease entity and social environment in which patients live. We developed a CU-specific QOL (CU-QOL) questionnaire as a validated tool, which considers Korean culture and language [10].

This study was aimed at evaluating QOL impairment in CSU patients using our own CU-QOL questionnaire and the correlation between UAS and CU-QOL. Additionally, we sought to investigate possible predictors of impaired CU-QOL.

\section{METHODS}

\section{Subjects}

We enrolled 390 adult patients with CSU who were followed in the Allergy and Clinical Immunology Clinic at
Ajou University Hospital from March 2009 to December 2012. Inclusion criteria were age $\geq 20$ years old and suffering from urticaria symptoms (USs), such as wheals and itching, for more than 6 weeks. Patients with urticarial vasculitis, physically inducible urticaria, or other chronic skin diseases were excluded.

\section{Assessment of the QOL (CU-QOL) and UAS}

The QOL was assessed with the CU-QOL questionnaire (range, o to 100), which has been shown to be a reliable and valid instrument. It consists of 17 items in four categories: US, emotional distress (ED), stigma, and food/ environmental distress (FE) [10].

CU disease activity was assessed using the total UAS (UAS-15), which was measured using a combination of four characteristics of wheals: numbers (UAS-1: 0 , no wheals; $1,<20$ wheals; 2,21 to 50 wheals; 3, > 50 wheals), distribution range (UAS-2: 0 , none; $1,<25 \%$ of the body surface area [BSA]; $2,25 \%$ to $50 \%$ of the BSA; $3,>50 \%$ of the BSA), mean diameter (UAS-3: 0 , no wheals; $1,<1 \mathrm{~cm}$; 2,1 to $3 \mathrm{~cm} ; 3,>3 \mathrm{~cm}$ ), and duration (UAS-4: o, no wheals; $1,<4$ hours; 2, 4 to 12 hours; $3,>12$ hours), and pruritus according to intensity (UAS-5: o, no pruritus; 1, mild; 2, moderate; 3, severe) within the last week for outpatient clinic visits, yielding a total score of o to $15[10,11]$. We also used the 6-point UAS (UAS-6), which is the sum of wheal numbers (UAS-1) and pruritus intensity (UAS-5) [2]. The presence of angioedema was also checked.

\section{Measurement of total immunoglobulin E}

Serum total immunoglobulin E (IgE) level was measured using the ImmunoCAP system (Pharmacia Diagnostics, Uppsala, Sweden) according to the manufacturer's instructions.

\section{Statistical analyses}

Data for continuous variable are shown as mean $\pm \mathrm{SD}$ or median values (minimum to maximum). Spearman's rho was used for the correlation of the CU-QOL and UAS items. A multivariate analysis was used for linear regression to determine predictors that influenced CUQOL. Logistic regression was used to evaluate the effects of various factors on CU-QOL. $p$ values $<0.05$ were considered to indicate statistical significance. All statistical analyses were performed using the SPSS version 12 (SPSS Inc., Chicago, IL, USA). 


\section{RESULTS}

\section{Clinical characteristics of the study subjects and assessment of CU-QOL and UAS}

In total, 390 Korean patients with CSU were enrolled. There were 158 men (40.5\%) and 232 women (59.5\%) with a mean age of $40.8 \pm 10.6$ years. The average CU duration was $32.6 \pm 52.1$ months. Urticaria was accompanied by angioedema in 120 of 308 patients (39.0\%). The mean UAS-15 was 10 (o to 15) and UAS-6 was 4 (o to 6). Mean $\log$ (total IgE) was $2.1 \pm 0.5$. The median CU-QOL score obtained from the questionnaire was 70.6 (o to 98.5). Patients were stratified into four quartiles based on total CU-QOL scores (Fig. 1). Patients possessing total CU-QOL scores below the fourth quartile (o to 85) were deemed to have CU-QOL impairment in this study. There were gradual and significant decreases in both UAS-15 and UAS-6 in the first through fourth quartiles but, otherwise, there were no significant differences in baseline demographic or clinical characteristics (Table 1). Additionally, significant variability was noted in the each of the four domains, when patients were classified by the quartile distribution of CU-QOL. The US domain score was the lowest, at 43.7 (o to 100) and the stigma domain score was as high as 100 (o to 100) among the four domains of CU-QOL (Table 1).

\section{Correlation between CU-QOL and UAS}

The total CU-QOL and each of the four category scores correlated significantly with the UAS, both UAS-15 and UAS-6. UAS-15 had a higher correlation (coefficient $-0.532, p<0.01)$ than UAS-6 $(-0.502, p<0.01)$. Each category of UAS had a higher correlation with the US and ED

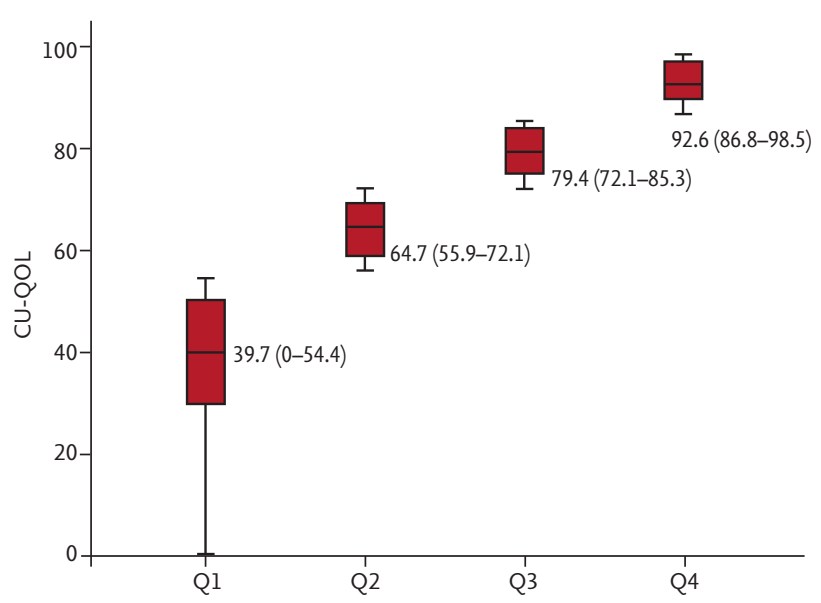

Figure 1. Interquartile distribution of chronic urticaria-specific quality of life (CU-QOL).

Table 1. Assessment of the CU-QOL and UAS

\begin{tabular}{|c|c|c|c|c|c|c|}
\hline Variable & Total $(n=390)$ & $Q_{1}(n=103)$ & $\mathrm{Q}_{2}(\mathrm{n}=98)$ & Q3 $(n=94)$ & $\mathrm{Q}_{4}(\mathrm{n}=95)$ & $p$ value \\
\hline Female sex & $232(59.5)$ & $57(55 \cdot 3)$ & $54(55.1)$ & $62(66.0)$ & $59(62.1)$ & $0.159^{\mathrm{a}}$ \\
\hline Age, yr & $40.8 \pm 10.6$ & $41.2 \pm 11.1$ & $39 \cdot 9 \pm 9 \cdot 5$ & $39.5 \pm 10.3$ & $42.5 \pm 11.2$ & $0.135^{b}$ \\
\hline Duration, mon & $32.6 \pm 52.1$ & $36.9 \pm 55.7$ & $31.4 \pm 44.7$ & $29.9 \pm 47.9$ & $31.8 \pm 58.9$ & $0.499^{b}$ \\
\hline UAS-15 & $10(0-15)$ & $12(0-15)$ & $11(0-15)$ & $10(0-15)$ & $5(0-15)$ & $<0.001^{b}$ \\
\hline UAS-6 & $4(0-6)$ & $5(0-6)$ & $4(0-6)$ & $4(0-6)$ & $2(0-6)$ & $<0.001^{b}$ \\
\hline Angioedema & $120 / 308(39.0)$ & $40(44.0)$ & $32(38.6)$ & $32(41.0)$ & $16(28.6)$ & $0.121^{a}$ \\
\hline $\log ($ total IgE) & $2.1 \pm 0.5$ & $2.2 \pm 0.4$ & $2.1 \pm 0.5$ & $2.0 \pm 0.6$ & $2.0 \pm 0.6$ & $0.143^{b}$ \\
\hline CU-QOL & $70.6(0-98.5)$ & $39.7(0-54.4)$ & $64.7(55.9-72.1)$ & $79.4(72.1-85.3)$ & $92.6(86.8-98.5)$ & $<0.001^{b}$ \\
\hline US domain & $43.7(0-100)$ & $18.7(0-93.7)$ & $31.2(0-100)$ & $56.2(0-100)$ & $93.7(50-100)$ & $<0.001^{b}$ \\
\hline ED domain & $70.0(0-100)$ & $30.0(0-60.0)$ & $60.0(30.0-100)$ & $85.0(35.0-100)$ & $95.0(70.0-100)$ & $<0.001^{b}$ \\
\hline FE domain & $75.0(0-100)$ & $33 \cdot 3(0-100)$ & $66.7(0-100)$ & $83.3(0-100)$ & $91.7(33.3-100)$ & $<0.001^{b}$ \\
\hline Stigma domain & $100(0-100)$ & $65.0(0-100)$ & $95.0(60.0-100)$ & $100(75.0-100)$ & $100(85.0-100)$ & $<0.001^{b}$ \\
\hline
\end{tabular}

Values are presented as number $(\%)$, mean $\pm \mathrm{SD}$, or median (minimum-maximum range).

CU-QOL, chronic urticaria-specific quality of life; UAS, urticaria activity score; Q, quartiles of CU-QOL; Log (total IgE), log-transformed serum total immunoglobulin E levels; US, current urticaria symptoms; ED, emotional distress; FE, food and environmental distress; Stigma domain, disgrace.

${ }^{\text {a }}$ Somers' D.

${ }^{\mathrm{b}}$ Kruskal-Wallis test. 
Table 2. Correlation between CU-QOL and UAS

\begin{tabular}{|c|c|c|c|c|c|c|c|}
\hline & UAS-15 & UAS-6 & UAS-1 & UAS-2 & UAS-3 & UAS-4 & UAS-5 \\
\hline Total CU-QOL & $-0.532^{\mathrm{a}}$ & $-0.502^{\mathrm{a}}$ & $-0.423^{\mathrm{a}}$ & $-0.487^{\mathrm{a}}$ & $-0.369^{\mathrm{a}}$ & $-0.404^{\mathrm{a}}$ & $-0.454^{\mathrm{a}}$ \\
\hline US domain & $-0.623^{a}$ & $-0.605^{\mathrm{a}}$ & $-0.45^{8^{a}}$ & $-0.55^{\mathrm{a}}$ & $-0.377^{\mathrm{a}}$ & $-0.498^{a}$ & $-0.593^{\mathrm{a}}$ \\
\hline ED domain & $-0.460^{a}$ & $-0.436^{\mathrm{a}}$ & $-0.388^{\mathrm{a}}$ & $-0.435^{\mathrm{a}}$ & $-0.332^{\mathrm{a}}$ & $-0.351^{\mathrm{a}}$ & $-0.377^{\mathrm{a}}$ \\
\hline FE domain & $-0.209^{a}$ & $-0.174^{a}$ & $-0.141^{a}$ & $-0.181^{a}$ & $-0.155^{\mathrm{a}}$ & $-0.143^{\mathrm{a}}$ & $-0.161^{a}$ \\
\hline Stigma domain & $-0.161^{a}$ & $-0.120^{b}$ & $-0.146^{\mathrm{a}}$ & $-0.156^{\mathrm{a}}$ & $-0.143^{\mathrm{a}}$ & $-0.100^{b}$ & -0.056 \\
\hline UAS-15 & & $0.905^{\mathrm{a}}$ & $0.817^{\mathrm{a}}$ & $0.832^{\mathrm{a}}$ & $0.793^{\mathrm{a}}$ & $0.800^{\mathrm{a}}$ & $0.787^{\mathrm{a}}$ \\
\hline UAS-6 & $0.905^{\mathrm{a}}$ & & $0.883^{\mathrm{a}}$ & $0.721^{\mathrm{a}}$ & $0.645^{\mathrm{a}}$ & $0.647^{\mathrm{a}}$ & $0.860^{a}$ \\
\hline
\end{tabular}

CU-QOL, chronic urticaria-specific quality of life; UAS, urticaria activity score; UAS-15, total score of UAS including UAS-1, $-2,-3,-4$, and -5; UAS-6, total score of UAS-1 and -5; UAS-1, the quantity of wheals; UAS-2, distribution ranges of wheals; UAS-3, mean wheal diameters; UAS-4, duration of wheals; UAS-5, intensity of pruritus within the last 1 week for outpatient clinic visit; US, current urticaria symptoms; ED, emotional distress; FE, food and environmental distress; Stigma domain, disgrace.

Significant (Spearman's rho) at ${ }^{\mathrm{a}} \mathrm{p}=0.01$ and ${ }^{\mathrm{b}} \mathrm{p}=0.05$, respectively.

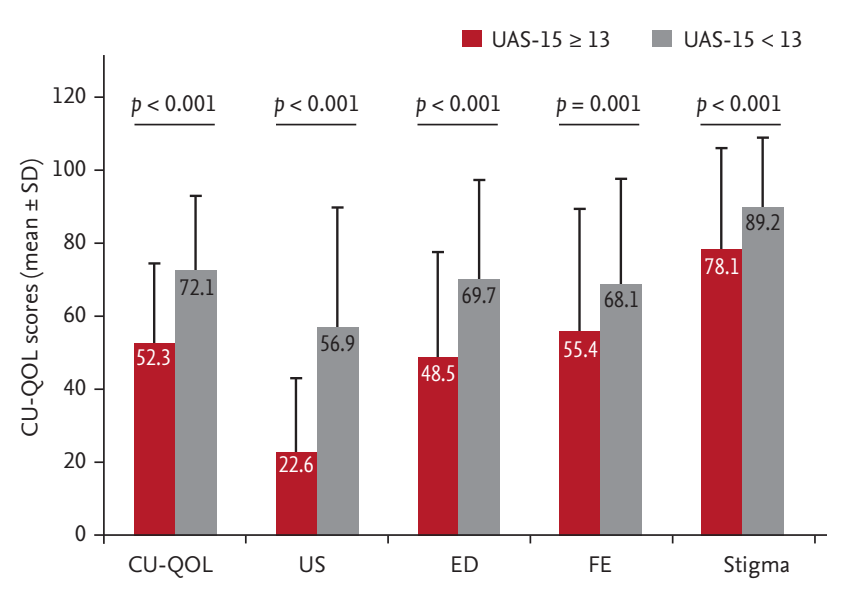

Figure 2. Comparison of chronic urticaria-specific quality of life (CU-QOL) between severe (urticaria activity score $[\mathrm{UAS}]-15 \geq 13)$ and non-severe CU (UAS-15 < 13). US, urticaria symptom domain; ED, emotional distress domain; FE, food and environmental stress domain.

domains of CU-QOL than the FE and stigma domains. UAS-2 (wheal distribution) had the highest negative correlation with total CU-QOL scores and each of the domains except the US domain, which correlated more strongly with UAS-5, the intensity of pruritus (Table 2).

\section{Predictors of CU-QOL}

We classified severe and non-severe CU according to a UAS-15 score of $\geq 13$, a determinant of uncontrolled CU in a previous study [11]. Total CU-QOL scores decreased significantly in patients with severe CU (52.3 vs. 72.1, p $<0.001$ ). Scores in each domain in CU-QOL were also significantly lower in severe CU than in non-severe CU

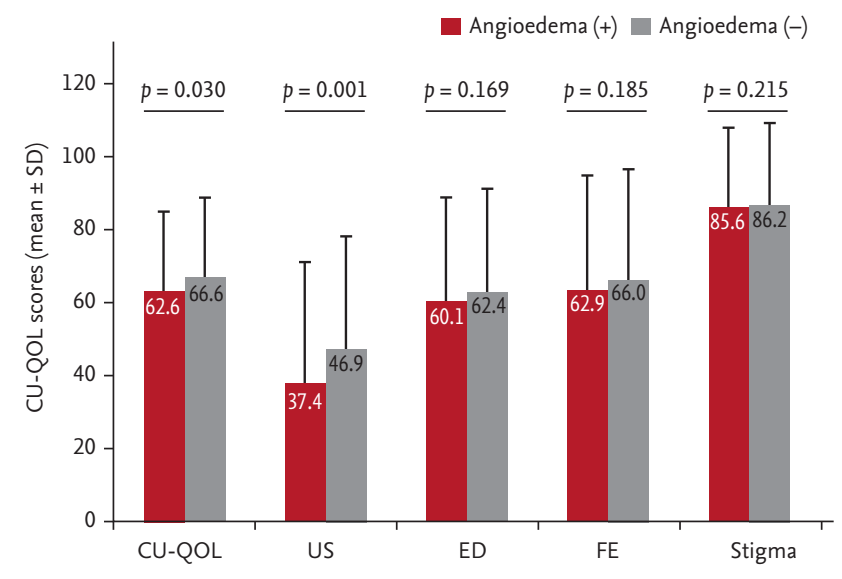

Figure 3. Comparison of chronic urticaria-specific quality of life (CU-QOL) according to the presence of angioedema. US, urticaria symptom domain; ED, emotional distress domain; FE, food and environmental stress domain.

patients (Fig. 2).

In comparing the CU-QOL scores according to the presence of angioedema, the US domain was significantly decreased in patients with angioedema compared with those with urticaria alone (37.4 vs. $46.9, p=0.004$ ). In contrast, there were no significant differences in total scores or the other three domains of CU-QOL between patients with and without angioedema (Fig. 3). Patients having angioedema also scored significantly higher values in both UAS-15 (10.0 \pm 4.1 vs. 8.9 $\pm 4.4, p=0.005)$ and UAS-6 $(4.0 \pm 1.8$ vs. $3.6 \pm 1.9, p=0.025)$ when compared to those without angioedema.

Log-transformed serum total IgE levels (log [total IgE]) 
Table 3. Multivariate analysis for predicting impaired chronic urticaria-specific quality of life $(<85)$

\begin{tabular}{lccc}
\hline Variable & $p$ value & Odds ratio & $95 \%$ CI \\
\hline Gender & 0.963 & 1.017 & $0.508-2.033$ \\
Age & 0.764 & 0.996 & $0.968-1.024$ \\
Urticaria duration & 0.225 & 0.997 & $0.992-1.002$ \\
UAS-15 $\geq 13$ & $<0.001$ & 9.371 & $2.798-31.383$ \\
Angioedema & 0.047 & 1.978 & $1.010-3.873$ \\
Log (total IgE) & 0.035 & 1.961 & $1.049-3.666$ \\
\hline
\end{tabular}

CI, confidence interval; UAS, urticaria activity score; Log (total IgE), log-transformed serum total immunoglobulin E levels (kU/L).

were significantly correlated with total CU-QOL (coefficient $-0.131, p<0.05)$ and ED domain scores $(-0.129, p<$ 0.05). A significant correlation of $\log$ (total IgE) was also noted with UAS-15 (0.123, $p=0.031)$, but not with UAS6 (0.019, $p=0.735)$. Regarding each category of UAS-15, UAS-2 (distribution range of wheals, coefficient $0.136, p$ $=0.017$ ) and UAS-3 (mean diameter of wheals, coefficient $0.211, p<0.001$ ) correlated significantly with log (total IgE), while the three other categories were unrelated to IgE levels.

A multivariate regression analysis revealed that severe CU (odds ratio [OR], 9.371; 95\% confidence interval [CI], 2.798 to $31.383 ; p<0.001$ ), $\log$ (total IgE) (OR, 1.961; 95\% CI, 1.049 to $3.666 ; p=0.035)$ and the presence of angioedema (OR, 1.978; 95\% CI, 1.010 to 3.873 ; $p=0.047$ ) were significant predictors of CU-QOL impairment after adjusting for gender, age, and urticaria duration (Table 3).

\section{DISCUSSION}

Recently, concerns about the deterioration of QOL of patients with $\mathrm{CU}$ have been heightened. In addition to clinical symptoms, like pruritus, wheals, and angioedema, many other factors, including the unexpectedness of symptom occurrence, sleep disturbances, and changes in appearance, are major problems for patients with CU. Thus, we need a more integrated approach to understand the whole influence of $\mathrm{CU}$ on patients. In the same context, recent guidelines and many health authorities have recommended using CU-QOL measurements and other patient-reported outcomes, like the urticaria control test [12], as major parameters in clinical practice and research [2,13].

The present study demonstrated substantial impair- ment in disease-specific QOL among patients with CSU. When patients were stratified into four quartiles, based on total CU-QOL scores, each of the four different dimensions also varied significantly. The US domain, which reflects on physical symptoms, was the most impacted domain. However, significant reductions in the ED and FE domains also suggest that CSU patients do not suffer only from physical symptoms but also from impairment in their emotional well-being and daily or social activities. Thus, understanding QOL in CSU patients is important to improve patient management.

In a previous study validating the CU-QOL in Korean patients, patients with CU as well as with physically inducible urticaria were enrolled. The main result from that study was that UAS, dermographism, and emotional stress were important predictors of CU-QOL in Korean CU patients [10]. The present study focused on measuring CU-QOL and exploring predictors of QOL impairment in patients with CSU. In addition to UAS15, the presence of angioedema and $\log$ (total IgE) were also identified as significant predictors of impaired CUQOL.

Most studies have reported that about 30\% to 50\% of CU patients have angioedema [10,14,15]. Toubi et al. [15] reported that more patients with accompanying angioedema were still suffering from urticaria at up to 5 years of follow-up compared with patients who exhibited only wheals ( $45 \%$ vs. $12 \%$ ). We found a significant association between co-existing angioedema and poor scores in the US domain of CU-QOL and both UAS15 and UAS-6. Although neither UAS-15 nor UAS-6, nor CU-QOL, consider angioedema sufficiently, more prominent disease activity and a significant difference in a specific QOL domain were noted in patients having angioedema, indicating that CU-QOL was a more reli- 
able measurement for Korean patients with CU. However, additional impairment in QOL in patients with CSU presenting with angioedema was not evident in a prior study using a different QOL measurement [16]. Thus, specific instruments to measure angioedema activity and angioedema-related QOL impairment in CU patients with recurrent angioedema with or without wheals have been proposed $[17,18]$.

Omalizumab (anti-IgE) is currently recommended for CU patients resistant to antihistamines, based on demonstrated efficacy and safety in recent clinical trials [19-21]. The importance of serum IgE levels in CU patients is increasingly recognized due to the clinical benefit of omalizumab, although it was effective in patients with CU regardless of IgE levels. Kessel et al. [22] reported that total serum IgE levels are frequently elevated in $\mathrm{CU}$ patients and these are significantly related to disease severity and duration. Increasing evidence suggests that autoreactivity including not only IgG autoantibodies against IgE and/or FceRI but also IgE antibodies to endogenous antigens, in patients with CSU plays an important role [23]. The IgE-occupied mast cells become more sensitive due to a decreased threshold for degranulation as well as those cells can store more mediators as compared with mast cells without IgE engagement [23]. A significant correlation between total IgE levels and urticaria disease activity was shown in the present study. However, $\log$ (total IgE) showed a significant correlation with UAS-15, but not with UAS-6. Log (total IgE) was associated significantly with the distribution range and mean diameter of wheals in CU patients, whereas we found no significant correlation between total IgE levels and the numbers of wheals and the intensity of pruritus. UAS- 6 does not consider other factors of urticaria disease activity, except wheal numbers and pruritus intensity. A sequential assessment of wheal numbers and pruritus every day for a week (UAS-7) has been adopted in recent guidelines and clinical research [2,19]. Despite a wider range (o to 42) of scores in UAS-7 compared with UAS-6 (o to 6), both UAS-7 and UAS-6 are limited in the evaluation of wheal numbers and pruritus. However, our results suggest that a more comprehensive assessment of the disease activity is appropriate for understanding the clinical characteristics of CU and for identifying major drivers of QOL impairment in CSU patients. We also found that log-transformed serum total IgE levels in pa- tients with CSU correlated significantly with total CUQOL scores and the ED domain. This relationship may be explained by the effect of $\operatorname{IgE}$ antibodies on the extent of mast cell activation and degranulation $[19,22]$. Checking the serum total IgE levels can be useful to predict urticaria severity and CU-QOL in patients with CSU.

Based on previous studies, the UAS, which combines wheal numbers and pruritus intensity, correlated significantly with QOL impairment, as measured using the Dermatology Life Quality Index in CU patients [24]. The present study showed the total CU-QOL scores and all individual domains correlated significantly with the UAS, and particularly well with the UAS-15, including duration, size, and distribution of wheals, when compared with the UAS-6. Of the five categories of UAS-15, UAS-2, which scores the distribution range of wheals, showed the strongest correlation with CU-QOL, but it is not included in UAS-6. Although the assessment of UAS- 15 requires more time than UAS-6, UAS- 15 is a more detailed and enhanced tool, and our results support that it is more suitable for the evaluation of impairment of CU-QOL.

We defined 'severe' CU as a UAS-15 score of 13 or more, as used previously [11]. A multivariate logistic regression demonstrated that severe CU was a significant and independent predictor of impaired CU-QOL, and it also correlated significantly with each domain factor. Regarding more severe CU leading to more frequently uncontrolled CU [11] and to a higher socio-economic burden [25], careful consideration of how serious QOL impairment is caused by CU is needed, particularly in patients with severe CU. We attempted to identify predictors of impaired CU-QOL in CSU patients. There have been many studies investigating QOL impairment in patients with CSU, but clinical interpretation according to QOL score is rare. A multivariate analysis showed that severe $\mathrm{CU}, \log$ (total IgE), and the presence of angioedema are significant predictors of impaired CU-QOL in Korean adults with CU.

In conclusion, physicians need to pay more attention to CU-QOL impairment in CSU patients for a better understanding of the disease burden in individual patients. Additionally, the assessment of patients in more comprehensive ways, including UAS-15, total IgE, and the presence of angioedema, to identify major determinants of CU-QOL is necessary. 


\section{KEY MESSAGE}

1. Chronic spontaneous urticaria (CSU) has a substantial negative impact on quality of life (QOL) of patients.

2. Severe chronic urticaria (CU), log (total immunoglobulin E), and the presence of angioedema are important predictors of impaired CU-QOL in Korean adults with CSU.

\section{Conflict of interest}

No potential conflict of interest relevant to this article was reported.

\section{Acknowledgments}

This work was supported by a Medical Research Center program (NRF-2012R1A5A2048183) through the National Research Foundation of Korea.

\section{REFERENCES}

1. Ye YM, Jang GC, Choi SH, et al. KAAACI Work Group report on the management of chronic urticaria. Allergy Asthma Respir Dis 2015;3:3-14.

2. Zuberbier T, Aberer W, Asero R, et al. The EAACI/GA(2) LEN/EDF/WAO guideline for the definition, classification, diagnosis, and management of urticaria: the 2013 revision and update. Allergy 2014;69:868-887.

3. Saini SS. Chronic spontaneous urticaria: etiology and pathogenesis. Immunol Allergy Clin North Am 2014;34:3352.

4. O'Donnell BF, Lawlor F, Simpson J, Morgan M, Greaves MW. The impact of chronic urticaria on the quality of life. Br J Dermatol 1997;136:197-201.

5. Engin B, Uguz F, Yilmaz E, Ozdemir M, Mevlitoglu I. The levels of depression, anxiety and quality of life in patients with chronic idiopathic urticaria. J Eur Acad Dermatol Venereol 2008;22:36-40.

6. Hashiro M, Okumura M. Anxiety, depression and psychosomatic symptoms in patients with atopic dermatitis: comparison with normal controls and among groups of different degrees of severity. J Dermatol Sci 1997;14:63-67.

7. Maurer M, Weller K, Bindslev-Jensen C, et al. Unmet clinical needs in chronic spontaneous urticaria: a GA(2)LEN task force report. Allergy 2011;66:317-330.

8. Ozkan M, Oflaz SB, Kocaman N, et al. Psychiatric morbidity and quality of life in patients with chronic idiopathic urticaria. Ann Allergy Asthma Immunol 2007;99:29-33.

9. Baiardini I, Pasquali M, Braido F, et al. A new tool to evaluate the impact of chronic urticaria on quality of life: chronic urticaria quality of life questionnaire (CU-QoL). Allergy 2005;60:1073-1078.

10. Ye YM, Park JW, Kim SH, et al. Clinical evaluation of the computerized chronic urticaria-specific quality of life questionnaire in Korean patients with chronic urticaria. Clin Exp Dermatol 2012;37:722-728.

11. Ye YM, Jin HJ, Hwang EK, et al. Co-existence of chronic urticaria and metabolic syndrome: clinical implications. Acta Derm Venereol 2013;93:156-160.

12. Weller K, Groffik A, Church MK, et al. Development and validation of the urticaria control test: a patient-reported outcome instrument for assessing urticaria control. J Allergy Clin Immunol 2014;133:1365-1372.

13. Baiardini I, Bousquet PJ, Brzoza Z, et al. Recommendations for assessing patient-reported outcomes and health-related quality of life in clinical trials on allergy: a GA(2)LEN taskforce position paper. Allergy 2010;65:290295 .

14. Zuberbier T, Balke M, Worm M, Edenharter G, Maurer M. Epidemiology of urticaria: a representative cross-sectional population survey. Clin Exp Dermatol 2010;35:869-873.

15. Toubi E, Kessel A, Avshovich N, et al. Clinical and laboratory parameters in predicting chronic urticaria duration: a prospective study of 139 patients. Allergy 2004;59:869873.

16. Poon E, Seed PT, Greaves MW, Kobza-Black A. The extent and nature of disability in different urticarial conditions. Br J Dermatol 1999;140:667-671.

17. Weller K, Groffik A, Magerl M, et al. Development and construct validation of the angioedema quality of life questionnaire. Allergy 2012;67:1289-1298.

18. Weller K, Groffik A, Magerl M, et al. Development, validation, and initial results of the angioedema activity score. Allergy 2013;68:1185-1192.

19. Maurer M, Rosen K, Hsieh HJ, et al. Omalizumab for the treatment of chronic idiopathic or spontaneous urticaria. N Engl J Med 2013;368:924-935.

20. Kaplan AP. Treatment of chronic spontaneous urticaria. Allergy Asthma Immunol Res 2012;4:326-331.

21. Nam YH, Kim JH, Jin HJ, et al. Effects of omalizumab 
treatment in patients with refractory chronic urticaria. Allergy Asthma Immunol Res 2012;4:357-361.

22. Kessel A, Helou W, Bamberger E, et al. Elevated serum total IgE: a potential marker for severe chronic urticaria. Int Arch Allergy Immunol 2010;153:288-293.

23. Chang TW, Chen C, Lin CJ, Metz M, Church MK, Maurer M. The potential pharmacologic mechanisms of omalizumab in patients with chronic spontaneous urticaria. J
Allergy Clin Immunol 2015;135:337-342.

24. Mlynek A, Zalewska-Janowska A, Martus P, Staubach P, Zuberbier T, Maurer M. How to assess disease activity in patients with chronic urticaria? Allergy 2008;63:777-780.

25. O'Donnell BF. Urticaria: impact on quality of life and economic cost. Immunol Allergy Clin North Am 2014;34:89104. 